\title{
Research on Data Transmission Method Based on 4G in Android
}

\section{Platform}

\author{
Yang Ningxia ${ }^{1}$, Chen Yongfeng ${ }^{2}$, Qin $\mathrm{Min}^{3}$ \\ ${ }^{1}$ Department of Yang Ningxia, Xi'an Communication Institute, 071000, China \\ ${ }^{2}$ Department of Chen Yongfeng, Agricultural University of Hebei, 071000, China \\ ${ }^{3}$ Department of Qin Min, Chongqing University of Posts and Telecommunications, 071000 , China \\ baodingynx@126.com
}

Keywords: 4G, Video data, Data transmission, Analysis of performance

\begin{abstract}
Aiming at studying the performance of the real-time video data transmission based on opportunistic network coding in the wireless network. First of all, making an in-depth analysis of the working principle of network coding and the improvement of the performance of the wireless network; secondly, on the basis of the brief introduction of the characteristics of video transmission to discuss the key points that the opportunistic network coding supporting the video transmission; finally, through the emulational method to study the performance of the real-time video transmission that based on opportunistic network coding in the wireless network, and compare and analyze the quality of the reconstructed image after transmission and the original image. The emulational results show that network coding can not only improve the throughput capacity of network, but also improve the quality of video transmission.
\end{abstract}

\section{Introduction}

In recent years, with the development of the communication network infrastructure, multimedia business occupies more and more important role in network communication. However, since the communication network can not provide reliable data transmission, especially the wireless network channel existing the characteristics of fading and interference will cause the information be affected by random errors and burst errors during the transmission, and leading to transmission errors of wrong code or data loss during the transmission of multimedia in the network, therefore, improving the quality of video transmission caused the extensive concern of the academia. At present, providing high quality video transmission in the wireless network environment is a challenging problem, the properties of unstabitily and time-variation of the wireless network making the video transmission have put forward higher requirements on the service quality of bandwidth, time delay and delay jitter and so on.

\section{The methods of video data transmission in $4 \mathrm{G}$}

\subsection{Opportunistic network coding (ONC)}

The core idea of ONC is that any node in the network can encode, transmit packets; each node saves three queues: Mixing-Q (store the coding packet that can encode), Q1 (store the packet that the destination node is this node ) and Q2 (store the packet that this node listening to), ONC using 
the strategy of multi priority data packet scheduling, giving higher sending priority to encoding packets; To achieve the effect of creating coding opportunities actively and effectively improve the throughput capacity of network.

ONC makes full use of those packets that listening to and being discarded in the traditional network protocol, and its working principle as shown in figure 1, in Figure 1 (a), according to the predefined path, the source node $\mathrm{X}$ will transmit node data packet P1 to the destination node $\mathrm{Y}$ through $\mathrm{A}$, the source node $\mathrm{U}$ needs to transmit data packet $\mathrm{P} 2$ to be transmitted to the destination node $\mathrm{V}$ through the $\mathrm{C}$ node. According to the characteristics of wireless information channel signal transmission, in the middle transmitting the competitive nodes B1, B2, and B3 can capture the data packages P1 and P2, and store them in the corresponding queues. In Figure 1 (b), B1, B2 and B3 nodes carry out the transmission by the method of competitive information channel according to the type of data packet in the queue, node B2 vied the information channel firstly and transmitted the encoding packet $\mathrm{P} 1 \oplus \mathrm{P} 2$, the node of the second hop $\mathrm{V}$ and $\mathrm{Y}$ receiving encoding package to successfully decode by using the data packets of the cache; and it will respond a ACK confirmation information.
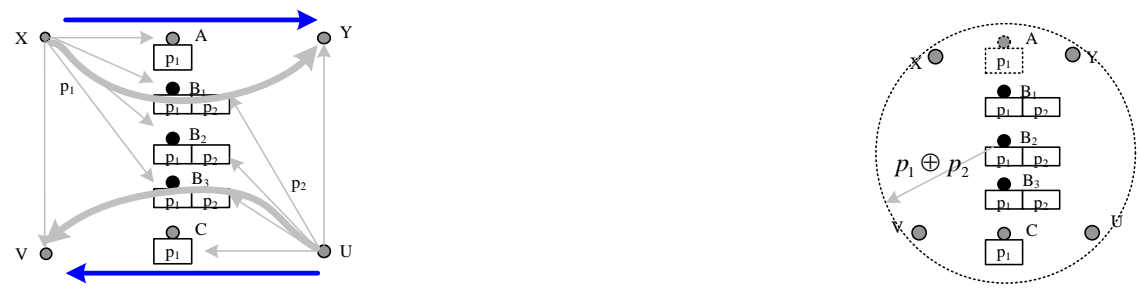

(a) Topological Structure (b) Selecting the prior and highest transmission encoding package Figure 1The working principle of the ONC protocol

\subsection{MPEG video transmission}

Video stream transmission is a real time communication technology, the original digital video stream transmission needs a huge channel capacity, so it is necessary to carry out video compression before the transmission. This paper defines three types of image (Frame) with considering the standard of the MPEG: (1) I-Frame(Intraframe), applied to the compression of its own data; (2)P-Frame (Uni- directional Prediction Frame)In decoding, it will refer to the previous I-Frame or P-Frame that is issued earlier; (3)B-Frame (Bi- direction Prediction Frame)In decoding, it will refer to the reference image data of two directions of the front or rear. Therefore, only when the receiving end receives all the relevant data frame, it can decode the received $\mathrm{P}$ frames and $\mathrm{B}$ frames. If an I-frame is lost or destroyed in the transmission process, the frame and all $\mathrm{P}$ frames and $\mathrm{B}$ frames relating with it will not be decoded, thus seriously affecting the quality of video stream transmission.

The performance of the network can be evaluated by using the throughput capacity, delay and delay jitter and other performance indexes; But the video stream transmission has particularity and the sensory of video image, so the evaluation of video transmission quality has become a very complex problem, it needs to consider the real-time and reliability. Generally, the most commonly used index of evaluation of the quality of video transmission is the peak signal to noise ratio (PSNR:Peak Signal to Noise Ratio) and the mean opinion score (MOS:Mean Opinion Score). PSNR is an objective video evaluation index that widely used in academic circles. Its core idea is to 
compare the original image $\mathrm{S}$ with the luminance part $\mathrm{Y}$ of the image $\mathrm{D}$, that is:

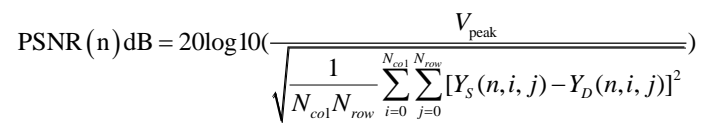

Among them, the $\mathrm{k}$ of Vpeak=2k-1 is the value of pixel that represented by a few bits for the brightness part, the bigger the value of PSNR, the smaller the difference between the objective image and the original image, that is, the quality of picture and video is better. MOS is a kind of subjective index that evaluating the quality of the digital image. The evaluation method is consistent with PSNR, and has a certain corresponding relation.

\section{The analysis of the experimental performance of the video transmission}

In this section, making sufficient analysis and research of the performance of real-time video transmission based on ONC wireless network by using the emulational method. Firstly, introducing the scene of simulation and the experimental design, and then the experimental results are analyzed and discussed.

In the environment of operating system Linux 10.4, on the emulational platform of NS2 achieved the transmission of video data based on ONC, and compared with 802.11 and the video transmission in COPE protocol, carrying out a comprehensive analysis and evaluation of the performance. Communication radius of nodes in the experiment is $250 \mathrm{~m}$, the transmission rate of channel data is $2 \mathrm{Mbps}$. The experiment selected YUV video files with QCIF format as the data source of node, the code is MPEG-4 format video sequence, sequence including 4 video streams with 300 frames (Foreman, Hall, Coastguard, Mother-, Daughter), the sending rate of video frame is $30 \mathrm{fps}$.

The experimental scenes used in this design include three level and cross topological structure. Cross topology as shown in Figure 2 (a), radius is $150 \mathrm{~m}$, each boundary node has 3 neighbor nodes, and intermediate nodes have 4 neighbor nodes, setting 2 couples of two two opposite streaming video in the network. In Figure 2 (b), the three level topology consists of three sets of nodes:Tier-1, Tier-2, Tier-3, Tier-1, Tier-2 are respectively composed of 4 nodes, Tier- 2 can be composed of 1 (2, 3 or 4 ) node. In Tier- 1 and Tier- 3 the nodes are the source node and the objective node, in Tier- 2 as a free node (that can participate in the competitive forwarding). Between Tier-2 and Tier-1, Tier- 3 only has a hop distance, interval is $200 \mathrm{~m}$, each node in the Tier-2 is able to capture all data packets sent by the source node and the objective node. Three level topology in this experiment having four kinds of topologies: 4-1-4, 4-2-4,4-3-4 and 4-4-4; the interception in figure was 4-2-4, i.e. Tier-2 has 2 nodes, Tier-1, Tier- 2 have 4 nodes. Two couples of the encoded video stream were set in the experiment of three level network topology.

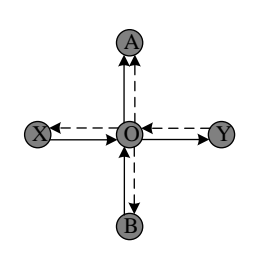

(a) cross topology

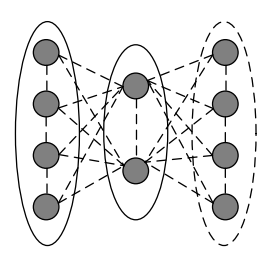

(b) three level topology

Figure 2. network topology structure

In this paper, designed two groups of experiments, respectively to simulate the performance of real-time video transmission that based on 802.11, COPE ONC protocol in the two topological structure, analyzing and comparing the solvable rate of data packets and packet loss rate, PSNR 
value and the video image restoration after the simulation.

Figure 2 shows the change of performance of receiver application layer in the four types of topology. It can be seen from the graph, the decodable frame rate of the four video fragments in topology of 4-1-4 is generally lower than the rest three kinds of structure, and the packet loss ratio is higher than the other three structures. Because in the structure of 4-1-4, Tier-2 only having one node, the congestion phenomenon will occur, resulting in the decrease of throughput capacity, packet loss rate will increase, which can also reduce the decoding frame rate.

With the 4-2-4 topology structure as an example, in Fig. 3 (a), the decodable frame rate of Foreman video clips is much higher than the decodable frame rate of the rest three video clips (Hall, Coastguard, Mother-Daughter); While in Figure 3 (b), we can see that the loss rate of I frame of Foreman video decreased a lot (compared to the rest of three video sequences); With Hall video segment as an example, in Fig. 3 (a), the decodable frame rate of Hall video fragments of 4-2-4 significantly improved a lot than the decodable frame rate of Hall video fragments of 4-3-4; As shown in Figure 3 (b), packet loss rate of I frame in Hall video clips of 4-2-4 significantly decreased a lot than in 4-3-4, but the change of the packet loss rate of B frame and P frame is not obvious at this time; At this time in Fig. 3 (a) we can see that the decodable frame rate of B, P, I-frames in the 4-2-4 compared with that in 4-3-4, having increased; the results can be seen from other video clips. This shows that the importance of I frames for the solvable rate of this video clip, we can improve the quality of video transmission by reducing the I frame loss rate.

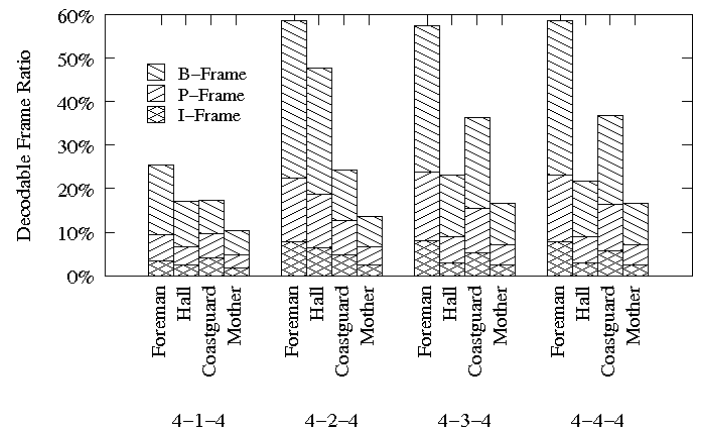

(a) Decodable frame rate

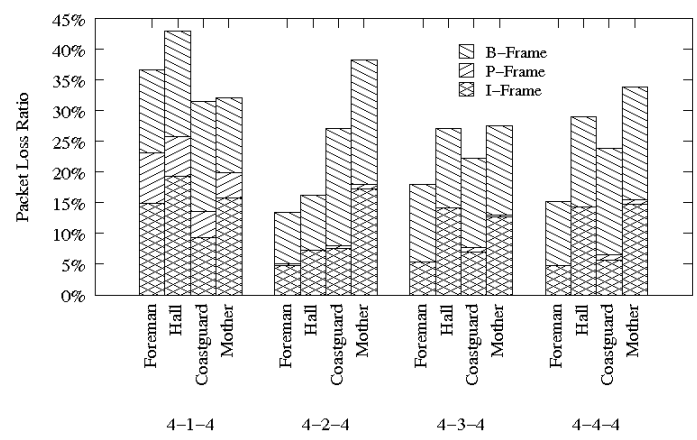

(b) Packet loss rate

Figure3 Three level topology

\section{Conclusion}

In this paper, we analyzed and studied the performance of real-time video transmission based on opportunistic network coding in wireless networks deeply, we designed two groups of experiments, to simulate the two sets of experiments by using NS2 simulation software, and comparing and analyzing the obtained reconstructed image and the obtained original image; Comparing and analyzing the decoding rate, packet loss rate in various cases, finding out the reasons of the decline of the decoding frame rate; Analyzing the effect of the loss of the three types of video frame, 
namely: the importance of the three video frames, which lays the foundation for the research work to improve the video transmission quality of the next step.

\section{Reference}

[1] J. He, Y. Geng and K. Pahlavan, Modeling Indoor TOA Ranging Error for Body Mounted Sensors, 2012 IEEE 23nd International Symposium on Personal Indoor and Mobile Radio Communications (PIMRC), Sydney, Australia Sep. 2012 (page 682-686)

[2] Y. Geng, J. Chen, K. Pahlavan, Motion detection using RF signals for the first responder in emergency operations: A PHASER project[C], 2013 IEEE 24nd International Symposium on Personal Indoor and Mobile Radio Communications (PIMRC), London,Britain Sep. 2013

[3] S. Li, Y. Geng, J. He, K. Pahlavan,Analysis of Three-dimensional Maximum Likelihood Algorithm for Capsule Endoscopy Localization, 2012 5th International Conference on Biomedical Engineering and Informatics (BMEI), Chongqing, China Oct. 2012 (page 721-725)

[4] Y. Geng, J. He, H. Deng and K. Pahlavan, Modeling the Effect of Human Body on TOA Ranging for Indoor Human Tracking with Wrist Mounted Sensor, 16th International Symposium on Wireless Personal Multimedia Communications (WPMC), Atlantic City, NJ, Jun. 2013.

[5] Y. Geng, J. He, K. Pahlavan, Modeling the Effect of Human Body on TOA Based Indoor Human Tracking[J], International Journal of Wireless Information Networks 20(4), 306-317 